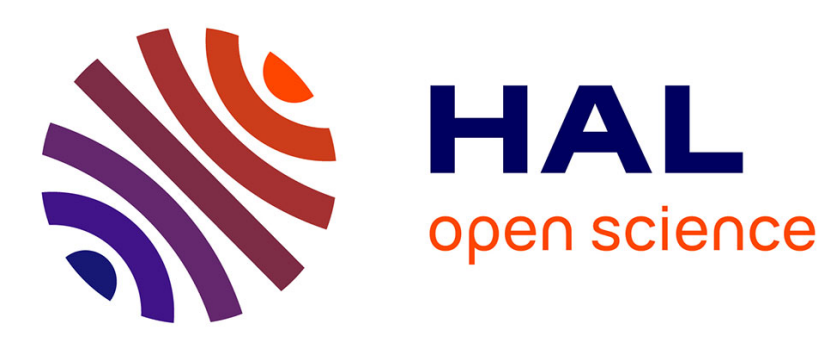

\title{
Estimation of time delays with fewer sensors than sources
}

\author{
Bruno Emile, Pierre Comon, Joel Le Roux
}

\section{To cite this version:}

Bruno Emile, Pierre Comon, Joel Le Roux. Estimation of time delays with fewer sensors than sources. IEEE Transactions on Signal Processing, 1998, 46 (7), pp.2012-2015. 10.1109/78.700972 . hal02100094

\section{HAL Id: hal-02100094 \\ https://hal.science/hal-02100094}

Submitted on 15 Apr 2019

HAL is a multi-disciplinary open access archive for the deposit and dissemination of scientific research documents, whether they are published or not. The documents may come from teaching and research institutions in France or abroad, or from public or private research centers.
L'archive ouverte pluridisciplinaire HAL, est destinée au dépôt et à la diffusion de documents scientifiques de niveau recherche, publiés ou non, émanant des établissements d'enseignement et de recherche français ou étrangers, des laboratoires publics ou privés. 


\section{Estimation of Time Delays with Fewer Sensors than Sources}

\author{
B. Emile, P. Comon, and J. Le Roux
}

\begin{abstract}
A number of papers have been dealing with the problem of estimating the differential delay of an unknown signal impinging on two sensors. The present contribution deals with the presence of more than one source, which is a case that has never been dealt with before. The solution resorts to slices of high-order spectra, and the full spectral band of the signals is utilized in order to recover the delays. It can be viewed as an improvement to the classical procedure consisting of searching the autocorrelation for local maxima, which does not work when delays are smaller than the source correlation length.
\end{abstract}

\section{INTRODUCTION}

It is assumed that $k$ real signals $s_{i}(t)$ are received on $l \leq k$ sensors. Those signals satisfy the equation model below (given here for $l=2$ ):

$r_{1}(t)=s_{1}(t)+s_{2}(t)+\cdots+s_{k}(t)+v_{1}(t)$,

$r_{2}(t)=s_{1}\left(t+\tau_{1}\right)+s_{2}\left(t+\tau_{2}\right)+\cdots+s_{k}\left(t+\tau_{k}\right)+v_{2}(t)$,

where $\tau_{i}$ denote delays, $v_{i}$ noises, and $s_{i}$ are unknown source signals. The problem consists of estimating delays $\tau_{i}$ from a finite extend observation. It is assumed that:

A1 The source signals are real and non Gaussian

A2 The source signals are mutually independent

A3 Delays $\tau_{i}$ are different

Note that assumption $\mathbf{A 3}$ is not restrictive, for if two delays $\tau_{i}$ and $\tau_{j}$ are equal, then sources $s_{i}$ and $s_{j}$ are undistinguishable. Thus it is assumed that nothing is known about the statistics of the sources but their non Gaussian character and their independence. In addition, because of the low SNR (Signal to Noise Ratio) in narrow band, it is necessary to fully take advantage of the signal bandwidth.

The identification of a differential delay between two signals is an old problem in signal processing; see for instance the June 1981 special issue of IEEE Transactions on ASSP. New methods have been proposed in [5], [14], [17] [11]. See also the approaches based on MUSIC-like algorithms [18] [15], with more sources than sensors [16] [3], based on the cyclostationarity of the source signals [10] or based on the knowledge of the steering vectors coefficients [19]. All these works are either dealing with the case of a single signal, i.e., $s_{2}=s_{3}=\ldots=s_{k}=0$, or take advantage of some knowledge about the array.

Some works have tackled blind identification of time delays in presence of more than one source (i.e. neither signals $s_{i}(t)$ nor their spectra are known, and the array is unknown), and include [4], [7] and [8]. But the appoach there is basically narrow-band, and there is always fewer signals than sensors.

Manuscript received Dec 2, 1996, revised Aug 30, and Oct 28, 1997. The associate editor coordinating the review of this paper and approving it for publication was Prof. Y. Hua. B. Emile is with ENSSAT, BP 447, 6 rue de Kérampont, F-22305 Lannion Cedex, P. Comon is with Eurecom Institute, BP 193, F-06904 Sophia-Antipolis cedex and with I3S-CNRS, and J. Leroux is with I3S-CNRS, 250 av Albert Einstein, F-06560 Sophia-Antipolis.
Recent techniques allow to virtually augment the size of the array, but localizing sources from such arrays can be seen as equivalent to applying a higher-order localization algorithm [6], e.g. 4-Music [3], or Virtual Esprit (Vespa) [12]. Note that previous works establishing bounds on the number of resolvable sources [1] are not questioned here since they hold true only in the Gaussian context.

In this article, we present a method for estimating delays between more source signals than sensors. Section III establishes the required equations where delays are the only unknowns in the spectral domain. Section IV solves the delay estimation problem in wide band.

\section{NOTATION}

In the spectral domain, denote the observations at pulsation $\omega:$

$$
\begin{aligned}
& r_{1}(\omega)=s_{1}(\omega)+s_{2}(\omega)+\cdots+s_{k}(\omega)+v_{1}(\omega), \\
& r_{2}(\omega)=s_{1}(\omega) x_{1}^{*}+s_{2}(\omega) x_{2}^{*}+\cdots+s_{k}(\omega) x_{k}^{*}+v_{2}(\omega) .
\end{aligned}
$$

where $x_{i}=e^{-\jmath \omega \tau_{i}}, \jmath=\sqrt{-1}$, and $\left(^{*}\right)$ denotes the complex conjugation. Define the following n-th order cumulant spectra of observations at the pulsation $\omega$ :

$$
\begin{gathered}
C_{i}^{(n)}=\operatorname{Cum}\{\underbrace{r_{1}(\omega), \cdots, r_{1}(\omega)}_{\frac{n}{2}}, \underbrace{r_{1}(-\omega), \cdots, r_{1}(-\omega)}_{\frac{n}{2}-i}, \\
\underbrace{r_{2}(-\omega), \cdots, r_{2}(-\omega)}_{i}\} .
\end{gathered}
$$

These spectra correspond to slices of the standard multivariate cumulant spectrum [2] [20] [13]. In this framework, $n$ must be even and $n \geq 2(k-1)$, where $k$ denotes the number of sources.

\section{PROBLEM FORMULATION}

\section{A. Preliminary basic properties}

The required equations are obtained by taking advantage of 3 basic properties, as shown below.

1) Independence property: Because of the independence between sources, the sensor cumulants $C_{i}^{(n)}$ can be written as:

$$
C_{i}^{(n)}=x_{1}^{i} \Gamma_{1}+\cdots+x_{k}^{i} \Gamma_{k},
$$

where $\Gamma_{p}$ are the sources cumulants:

$$
\Gamma_{p}=\operatorname{cum}\{\underbrace{s_{p}(\omega), \cdots, s_{p}(\omega), s_{p}(-\omega), \cdots, s_{p}(-\omega)}_{n}\} .
$$

Letting $i$ range in $\{0, \ldots, k-1\}$, the following system is satisfied:

$$
\left(\begin{array}{c}
C_{0}^{(n)} \\
C_{1}^{(n)} \\
\vdots \\
C_{k-1}^{(n)}
\end{array}\right)=\left(\begin{array}{cccc}
1 & 1 & \cdots & 1 \\
x_{1} & x_{2} & \cdots & x_{k} \\
\vdots & \vdots & & \vdots \\
x_{1}^{k-1} & x_{2}^{k-1} & \cdots & x_{k}^{k-1}
\end{array}\right)\left(\begin{array}{c}
\Gamma_{1} \\
\Gamma_{2} \\
\vdots \\
\Gamma_{k}
\end{array}\right) .
$$

In compact notation, the last relation can be rewritten as follows:

$$
C=V \Gamma \quad(\text { property } I) .
$$


The above relation involves $2 k$ unknowns, but only $k$ equations. Therefore, the identification of these $2 k$ parameters cannot be carried out by a technique such as the one described in [11] or in references therein.

2) Van der Monde property: Let $V$ be a Van der Monde matrix, as the one defined in equation (5), and $P_{i}$ be a symmetric polynomial of degree $i$ in $k$ variables defined as: $P_{0}=1, P_{1}=$ $x_{1}+x_{2}+\cdots+x_{k}, P_{2}=x_{1} x_{2}+x_{1} x_{3}+\cdots+x_{k-1} x_{k}, \cdots, P_{k}=$ $x_{1} x_{2} \cdots x_{k}$. If $Q^{T}=\left[(-1)^{k-1} P_{k-1}, \cdots,-P_{1}, P_{0}\right]$, then the following property is obtained:

$$
\left.Q^{T} V=(-1)^{k-1} X^{T}, \quad \text { (property } I I\right)
$$

where $X^{T}=\left[x_{2} \cdots x_{k}, x_{1} x_{3} \cdots x_{k}, \cdots, x_{1} \cdots x_{k-1}\right]$. In other words, the sum of the components of $X$ is the first entry of $Q$, up to a sign.

3) Unit modulus property: The complex conjugate of $C_{1}^{(n)}$ can be written as: $C_{1}^{(n) *}=x_{1}^{*} \Gamma_{1}+\cdots+x_{k}^{*} \Gamma_{k}$. Now multiply both sides of the previous equation by $P_{k}$ and use the fact that for all $i \in\{1, \ldots, k\},\left|x_{i}\right|=1$ since $x_{i}=e^{-\jmath \omega \tau_{i}}$, we obtain: $P_{k} C_{1}^{(n) *}=x_{2} \cdots x_{k} \Gamma_{1}+\cdots+x_{1} \cdots x_{k-1} \Gamma_{k}$. Or with the previous compact notation:

$$
P_{k} C_{1}^{(n) *}=X^{T} \Gamma \quad(\text { property } I I I) .
$$

\section{B. Results}

With the help of the three properties above, the unknown source cumulants $(\Gamma)$ can be eliminated:

$$
\begin{aligned}
Q^{T} C & =Q^{T} V \Gamma, & & \text { from }(I) \\
& =(-1)^{k-1} X^{T} \Gamma, & & \text { from }(I I) \\
& =(-1)^{k-1} C_{1}^{(n) *} P_{k} . & & \text { from }(I I I)
\end{aligned}
$$

Equation (8) then yields:

$$
\sum_{i=0}^{k-1}(-1)^{i} P_{i} C_{k-1-i}^{(n)}=(-1)^{k-1} C_{1}^{(n) *} P_{k},
$$

where $C_{i}^{(n)}$ can be estimated (cross-cumulants between the sensors), and where the $P_{i}$ 's contain the unknown delay information.

\section{ESTIMATION OF DELAYS}

Equation (9) can be arranged as follows:

$$
\frac{C_{k-1}^{(n)}}{C_{k-2}^{(n)}}=P_{1}-\frac{1}{C_{k-2}^{(n)}}\left(\sum_{i=2}^{k-1}(-1)^{i} P_{i} C_{k-1-i}^{(n)}+(-1)^{k} C_{1}^{(n) *} P_{k}\right)
$$

In $P_{i}$, all delays are represented by variables $x_{j}=e^{-\jmath \omega \tau_{j}}$. Now, if we take the inverse Fourier transform of (10), we obtain $k$ peaks, each representing one delay (the $P_{1}$ term), and several attenuated peaks located at partial sums of the delays (terms $P_{i}, i \neq 1$ ). If the number of delays is known, it is then sufficient to estimate the location of the first $k$ peaks, that represent the delays $\tau_{j}$.

Equation (10) can be computed for every pulsation $\omega$ such that $C_{k-2}^{(n)}(\omega) \neq 0$ in the signal bandwidth.


Fig. 1. Inverse Fourier transform of $C_{1}^{(2)} / C_{0}^{(2)}$ (top), and of $C_{1}^{(2)}$ (botton), $k=2$ sources, $\tau_{1}=2.3, \tau_{2}=4.2$ (interpolated zoom on the first 20 frequency bins, after a FT of length 256).

1) Example: $k=2$ and $n \geq 2$ : If $n=2$ is chosen, the following equation is obtained:

$$
C_{1}^{(2)}=P_{1} C_{0}^{(2)}-P_{2} C_{1}^{(2) *} .
$$

Since $P_{1}=x_{1}+x_{2}$, the inverse Fourier transform of $P_{1}$ gives two peaks at $\tau_{1}$ and $\tau_{2}$. As shown in Figure 1 by taking the inverse Fourier transform of $C_{1}^{(2)} / C_{0}^{(2)}$, we find two peaks and an attenuated peak at $\tau_{1}+\tau_{2}\left(P_{2}=x_{1} x_{2}\right)$. In the bottom of Figure 1, the plot of the raw cross correlation $C_{1}^{(2)}$ shows that the delays cannot be detected because the correlation length of the sources is too long. If $n=4$, the same equation would be constructed.

2) Example: $k=3$ and $n \geq 3$ : Since $n$ must be even, the smallest $n$ we can consider is $n=4$. The following equation is obtained:

$$
C_{2}^{(4)}=P_{1} C_{1}^{(4)}-P_{2} C_{0}^{(4)}+P_{3} C_{1}^{(4) *}
$$

The inverse Fourier transform of $C_{2}^{(4)} / C_{1}^{(4)}$ gives three peaks, at $\tau_{1}, \tau_{2}$ and $\tau_{3}$, and attenuated peaks at $\tau_{1}+\tau_{2}, \tau_{1}+\tau_{3}, \tau_{2}+\tau_{3}$, and $\tau_{1}+\tau_{2}+\tau_{3}$, as shown in Figure 2 .

3) Limitations: The proposed method has some restrictions: (i) If a peak corresponding to a delay is too close to another one corresponding to the partial sum of delays, then the identification becomes ill-conditionned.

(ii) Obviously, if delays are too close to each other, a single peak might be detected.

(iii) Because of the relation between the number of sources 


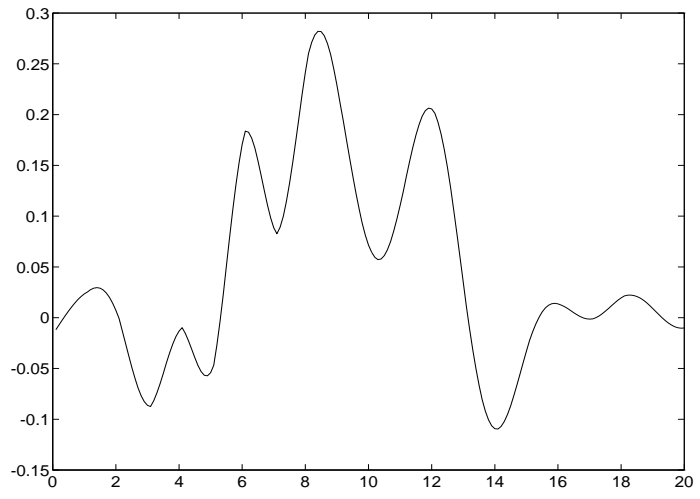

Fig. 2. Inverse Fourier transform of $C_{2}^{(4)} / C_{1}^{(4)}, k=3$ sources, $\tau_{1}=$ $6.2, \tau_{2}=8.6, \tau_{3}=11.9$ (interpolated zoom on the first 20 frequency bins, after a FT of length 256).

and the order of cumulants $n \geq 2(k-1)$, only three source signals can be considered if fourth order cumulants are used. (iv) It is useful to know the number of source signals, especially when it is difficult to differentiate between peaks corresponding to delays and those corresponding to sum of delays.

If delays are well separated (compared to source correlation length), a mere maxima search of the autocorrelation function can be sufficient. This method yields a solution when delays are separated by a gap that is much smaller than the correlation length of the signal. It can be applied to several problems in Sonar, Radar, or telecommunications.

\begin{tabular}{|l|l|l||l|l|}
\cline { 2 - 5 } \multicolumn{1}{c|}{} & \multicolumn{2}{c||}{$\tau_{1}$} & \multicolumn{2}{c|}{$\tau_{2}$} \\
\cline { 2 - 5 } \multicolumn{1}{c|}{} & mean & std & mean & std \\
\hline M1 & 2.16 & 0.014 & 4.1 & 0.041 \\
M2 & 2.30 & 0.047 & 4.19 & 0.004 \\
\hline
\end{tabular}

MEAN AND STANDARD DEVIATION (STD) OF ESTIMATED DELAYS OVER 100 INDEPENDENT TRIALS USING THE WIDE-BAND SPECTRAL APPROACH (M1) AND THE TIME DOMAIN APPROACH (M2). TRUE DELAYS ARE 2.3 AND 4.2 IN THIS SIMULATION.

\section{Simulation Results}

The signals $s_{i}(t)$ are ARMA processes driven by a i.i.d. sequence uniformly distributed with zero mean and unit variance: $s_{i}(t)=-a_{1, i} s_{i}(t-1)-a_{2, i} s_{i}(t-2)+v_{i}(t)+$ $b_{1, i} l_{i}(t-1)+b_{2, i} l_{i}(t-2)$. Coefficients are defined as: $a_{1, i}=-2 \rho_{i} \cos \theta_{i}, a_{2, i}=\rho_{i}^{2}, b_{1, i}=-2 \lambda_{i} \cos \phi_{i}, b_{2, i}=\lambda_{i}^{2}$ and $\theta_{1}=60^{\circ}, \theta_{2}=30^{\circ}, \theta_{3}=40^{\circ}, \rho_{1}=0.7, \rho_{2}=0.8$, $\rho_{3}=0.6, \phi_{1}=110^{\circ}, \phi_{2}=140^{\circ}, \phi_{3}=160^{\circ}, \lambda_{1}=0.8$, $\lambda_{2}=0.9, \lambda_{3}=0.7$.

All results are obtained over 100 independent trials, each of sample size 10000. Table I summarizes the results with two delays (without noise). The method M1 is the one described in section IV-1. The inverse Fourier transform of $\left(C_{1}^{(2)} / C_{0}^{(2)}\right)$ is interpolated with the cardinal sine function in order to find the maxima of the function with increased accuracy. The method M2 is the optimization method described in [9], with initial guesses given by method M1.

The advantage of the method M1 is that it does not need initial guesses, and that it is wide-band, compared to the

\begin{tabular}{|c|c||c|c|c|c|}
\cline { 2 - 6 } \multicolumn{1}{c|}{} & \multicolumn{2}{c||}{ SNR } & \multicolumn{2}{c|}{$\tau_{1}$} & \multicolumn{2}{c|}{$\tau_{2}$} \\
\cline { 3 - 6 } \multicolumn{1}{c|}{} & $(\mathrm{dB})$ & mean & std & mean & std \\
\hline$M_{1}$ & 0 & 4.01 & 0.20 & 7.98 & 0.62 \\
$M_{2}$ & 0 & 3.28 & 0.86 & 9.38 & 1.66 \\
\hline$M_{1}$ & 10 & 3.33 & 0.22 & 4.04 & 0.20 \\
$M_{2}$ & 10 & 3.56 & 0.27 & 4.02 & 0.36 \\
\hline$M_{1}$ & 12 & 2.16 & 0.02 & 4.06 & 0.07 \\
$M_{2}$ & 12 & 2.31 & 0.05 & 4.21 & 0.03 \\
\hline
\end{tabular}

MEAN AND STANDARD DEVIATION OF ESTIMATED DELAYS OVER 100 INDEPENDENT TRIALS WITHOUT ATTENUATIONS USING THE WIDE-BAND SPECTRAL APPROACH (M1) AND THE TIME DOMAIN APPROACH (M2) IN A NOISY CONTEXT. TRUE DELAYS ARE 2.3 AND 4.2 IN THIS SIMULATION.

\begin{tabular}{|l|l|l||l|l||l|l|}
\cline { 2 - 7 } \multicolumn{1}{c|}{} & \multicolumn{2}{c||}{$\tau_{1}$} & \multicolumn{2}{c||}{$\tau_{2}$} & \multicolumn{2}{c|}{$\tau_{3}$} \\
\cline { 2 - 7 } \multicolumn{1}{c|}{} & mean & std & mean & std & mean & std \\
\hline M1 & 6.1 & 0.016 & 8.42 & 0.039 & 11.95 & 0.05 \\
\hline
\end{tabular}

MEAN AND STANDARD DEVIATION (STD) OF ESTIMATED DELAYS OVER 100 INDEPENDANT TRIALS USING THE SPECTRAL METHOD WITH 2 SENSORS AND 3 SOURCE SIGNALS (M1). TRUE DELAYS ARE 6.2, 8.6 AND 11.9 IN THIS SIMULATION.

spectral method proposed in [8]. The time domain optimization improves the result.

The same approach (table I) is presented with independent noises $v_{1}$ and $v_{2}$. The numerical value of delays has been chosen in order to find the limit of validity of the approach. The signal to noise ratio $(S N R)$ is defined as $S N R=10 \log \left(\operatorname{std}\left(s_{1}+s_{2}\right) / \operatorname{std}\left(v_{1}\right)\right)$, where std denotes standard deviation.

The limit of performance is reached when the two peaks cannot be separated (about $S N R=12 d B$ ). With $S N R=$ $0 d B$, the second peak detected is located in the neighborhood of the sum of the two delays (without noise), which explains the bias. Table III presents the wide-band method described in section IV-2 with three delays.

This result is attractive, because with only two sensors, it is possible to estimate the delays of three source signals using fully the signal bandwidth.

\section{CONCLUSION}

The algorithm described in this paper allows the estimation of relative differential delays between more sources than sensors, in a wide-band context. It can also be seen as a whitening operation applicable when sources are unknown, because of the division by $C_{k-2}^{(n)}$. This key operation strongly increases accuracy. For the moment, the algorithm cannot be compared to others, since none exists that is able to perform blind identification of time delays when the number of sensors is not larger than the number of sources. Following the same lines as in [9], unknown attenuations can be taken into account as well.

\section{REFERENCES}

[1] Y. BRESLER, "On the resolution capacity of wideband sensor arrays: Further results", in Proc. ICASSP, Toronto, May 1991, pp. 1353-1356.

[2] D. R. BRILLINGER, Time Series, Data Analysis and Theory, HoldenDay, 1981.

[3] J. F. CARDOSO, "How much more DOA information in higher order statistics ?", in Proc. 7th workshop on SSAP, Quebec City, 1994, pp. 199-202. 
[4] J. F. CARDOSO, A. SOULOUMIAC, "Blind beamforming for nonGaussian signals", IEE Proceedings - Part F, vol. 140, no. 6, pp. 362 370, Dec. 1993, Special issue on Applications of High-Order Statistics.

[5] Y.T. CHAN, J.M. RILEY, J.B. PLANT, "A parameter estimation approach to time-delay estimation and signal detection", IEEE Trans. ASSP, vol. 28, no. 1, pp. 8-16, Febrary 1980.

[6] P. CHEVALIER, "New geometrical results about fourth-order direction finding method performance", in EUSIPCO 96, Trieste, Italy, September 10-13 1996, pp. 923-926.

[7] P. COMON, "Independent Component Analysis, a new concept ?", Signal Processing, Elsevier, vol. 36, no. 3, pp. 287-314, Apr. 1994, Special issue on Higher-Order Statistics.

[8] P. COMON, B. EMILE, "Estimation of time delays in the blind mixture problem", in Proc. EUSIPCO, Edinburgh, Scotland, 1994, pp. 482-485.

[9] B. EMILE, P. COMON, J. LEROUX, "Estimation of time delays between wide-band sources", in Proc. of the IEEE-ATHOS workshop on High-Order Statistics, Begur, Girona, SPAIN, June 1995, pp. 111-115.

[10] W.A. GARDNER, C.K. CHEN, "Signal-selective time-difference-of arrival estimation for passive location of man-made signal sources in highly corruptive environments, part1: theory and method", IEEE Trans. Sig. proc., vol. 40, no. 5, pp. 1168-1184, May 1992.

[11] Y. HUA, T. K. SARKAR, "Parameter estimation of multiple transient signals", Signal Processing, vol. 28, pp. 109-115, 1992.

[12] J. MENDEL, M. C. DOGAN, "Higher-order statistics applied to some array signal processing problems", in IFAC-SYSID, 10th IFAC Symposium on System Identification, M. Blanke, T. Soderstrom, Eds., Copenhagen, Denmark, July 4-6 1994, vol. 1, pp. 101-106, invited session.

[13] C. L. NIKIAS, A. P. PETROPULU, Higher-Order Spectra Analysis, Signal Processing Series. Prentice-Hall, Englewood Cliffs, 1993.

[14] C.L. NIKIAS, R.PAN, "Time delay estimation in unknown spatially correlated noise.", IEEE Trans. ASSP, vol. 36, no. 11, pp. 1706-1714, November 1988.

[15] M.A. PALLAS, G. JOURDAIN, "Active high resolution time delay estimation for large BT signals.", IEEE Trans. Sig. Proc., vol. 39, no. 4, pp. 781-188, April 1991.

[16] S. SHAMSUNDER, G.B. GIANNAKIS, "Modeling of non-gaussian array data using cumulants: DOA estimation of more sources with less sensors", Signal Processing, vol. 30, pp. 279-297, 1993.

[17] J. TUGNAIT, "On time delay estimation with unknown spatially correlated Gaussian noise using fourth-order cumulants", IEEE Trans. Sig. proc., vol. 36, no. 9, pp. 1258-1267, June 1991.

[18] G. VEZZOSI, "Estimation of phase angles from the cross-spectral matrix.", IEEE Trans. ASSP, vol. 34, no. 3, pp. 405-422, June 1986.

[19] M. WAX, J. SHEINVALD, J. WEISS, "Detection and localisation in colored noise via generalized least squares", IEEE Trans. Sig. Proc., vol. 44, no. 7, pp. 1734-1743, July 1996.

[20] I. G. ZURBENKO, The spectral analysis of time series, North-Holland, 1985. 